Journal Club

Editor's Note: These short, critical reviews of recent papers in the Journal, written exclusively by graduate students or postdoctoral fellows, are intended to summarize the important findings of the paper and provide additional insight and commentary. For more information on the format and purpose of the Journal Club, please see http://www.jneurosci.org/misc/ifa_features.shtml.

\title{
Strengthening Corticospinal Connections with Chronic Electrical Stimulation after Injury
}

\author{
Alain Frigon, Sergiy Yakovenko, Valeriya Gritsenko, Marie-Ève Tremblay, and Gregory Barrière \\ Groupe de Recherche sur le Système Nerveux Central, Faculté de Médecine, Université de Montréal, Montréal, Québec, Canada H3T 1J4 \\ Review of Brus-Ramer et al. (http://www.jneurosci.org/cgi/content/full/27/50/13793)
}

Damage to descending tracts from supraspinal structures impairs motor function below the level of the lesion, and efforts are being made to elucidate the mechanisms promoting the recovery process. One approach to facilitate motor recovery after neurotrauma is to target intact or spared pathways. For example, in a recent study published in The Journal of Neuroscience, spared corticospinal axons were chronically stimulated to strengthen their connections with spinal circuits in adult rats (Brus-Ramer et al., 2007). Results indicated that activity-dependent mechanisms facilitate plasticity of spared ipsilateral corticospinal axons after injury.

In the study by Brus-Ramer et al. (2007), the left pyramidal tract of adult rats was lesioned, abolishing corticospinal projections to the ipsilateral and contralateral spinal cord. In some rats, an electrode was placed over the right pyramidal tract to chronically stimulate spared corticospinal axons after lesion. The intact ipsilateral corticospinal tract was subsequently examined using electrophysiological and anatomical techniques. Comparisons were made between intact rats, rats with lesion only, rats with corti-

Received Jan. 23, 2008; revised Feb. 21, 2008; accepted Feb. 21, 2008 Correspondence should be addressed to Alain Frigon, Department of Physiology, Faculty of Medicine, Université de Montréal, Pavillon Paul-G.Desmarais, 2960, chemin de la Tour, Montréal, Québec, Canada H3T 1 J4. E-mail: alain.frigon@umontreal.ca.

DOI:10.1523/JNEUROSCI.0308-08.2008

Copyright $\odot$ 2008 Society for Neuroscience $\quad$ 0270-6474/08/283262-02\$15.00/0 cospinal stimulation only, and lesioned rats that underwent stimulation.

To compare the extent of axon collateral sprouting and synapse formation between groups, the anterograde tracers biotinylated dextran amine (BDA) and Lucifer yellow-dextran amine were injected into "spared" and "lesioned" motor cortices, respectively, 2 weeks before the pyramidal tract lesion. The authors found that total axon length, density of terminations, and boutons from spared ipsilateral corticospinal axons increased in the cervical spinal gray matter with injury or stimulation alone, but the combined lesionplus-stimulation group displayed the largest effect [Brus-Ramer et al. (2007), their Fig. 3 (http://www.jneurosci.org/ cgi/content/full/27/50/13793/F3)]. A ventral shift in the topographic distribution of the terminations and boutons from spared ipsilateral corticospinal axons was evident [Brus-Ramer et al. (2007), their Fig. 4 (http://www.jneurosci.org/cgi/ content/full/27/50/13793/F4)] in all experimental groups, suggesting that more direct synaptic contacts were made with motoneurons.

High-resolution electron microscopy would be necessary to show conclusively what neurons the spared ipsilateral corticospinal axons synapse onto. Because BDA labeling is revealed with diaminobenzidine, which precipitates into an electron-dense reaction product, the BDA-labeled ipsilateral corticospinal presynaptic terminals could be visualized di- rectly and their postsynaptic partners identified using retrograde tracers, immunocytochemical markers, or morphological criteria. The relative proportions of ipsilateral corticospinal presynaptic terminals contacting different types of postsynaptic partners could be determined and compared between groups. More direct synaptic contacts with motoneurons could indicate that spared ipsilateral corticospinal axons are attempting to form new and more direct spinal motor circuits.

To determine whether structural changes correlated with an increased transmission efficacy of spared corticospinal axons, Brus-Ramer et al. (2007) stimulated the intact pyramid and recorded the electroneurogram of the deep radial nerves. This allowed them to determine the motor thresholds (that is, the threshold of stimulation required to evoke a response in the nerve) of intact ipsilateral and contralateral corticospinal projections in all groups of rats. In control animals, the ratio between the ipsilateral and contralateral projections was relatively high because the efficacy of the contralateral corticospinal projection is much greater (i.e., has a lower motor threshold) than that of the ipsilateral projection. This is partly because of denser contralateral projections. In contrast, the ratio of motor thresholds between the intact ipsilateral and contralateral corticospinal projections was decreased in injury-alone and stimulation-alone groups. However, the 
largest reduction was observed in the combined injury and stimulation group, which the authors interpreted as indicative of increased transmission efficacy of spared ipsilateral corticospinal axons [Brus-Ramer et al. (2007), their Fig. 1 B, C (http://www.jneurosci.org/cgi/content/ full/27/50/13793/F1)]. In other words, the spared ipsilateral corticospinal projection assumes more control after the loss of the contralateral projection from the lesion side. However, whether decreased ratios resulted from increased ipsilateral efficacy, decreased contralateral efficacy, or both is unclear. To more clearly show that ipsilateral efficacy was increased after the lesion and/or with stimulation, some absolute values of motor thresholds bilaterally would have to be provided. Another option to further strengthen the authors' conclusions would be chronic implantations of nerve cuff and intramuscular electrodes to record pyramidal tract-evoked potentials in the same animal before and after the lesion.

Although Brus-Ramer et al. (2007) found a correlation between structural changes and supposed increased ipsilateral corticospinal efficacy, it is unclear whether new connections were functional. Increased ipsilateral efficacy could simply result from disinhibition at a spinal level. The contralateral corticospinal tract probably contacts several inhibitory and excitatory interneurons that in turn influence the excitability of ipsilateral pathways (Schomburg, 1990). Consequently, removing contralateral corticospinal axons would shift the balance between excitation and inhibition, to favor excitation. The resulting disinhibition of the ipsilateral corticospinal tract would thereby increase its efficacy. Whether supraspinal structures and/or the spinal cord mediate changes in the efficacy of the ipsilateral corticospinal tract should be investigated in the future.

The functional significance of the structural and physiological changes described by Brus-Ramer et al. (2007) requires investigation. For instance, increased efficacy of spared corticospinal tracts could improve performance of skilled movements, which could be assessed by specialized tests, such as reaching and grasping a reward or the ladderrung walking test (Whishaw and Metz, 2002). Additionally, enhanced axonal sprouting and varicosities in dorsal layers [Brus-Ramer et al. (2007), their Fig. 2 (http://www.jneurosci.org/cgi/content/ full/27/50/13793/F2)] might increase nociception. Increased sensitivity of sensory, particularly pain-relaying pathways could be tested with standardized withdrawal reflex tests, such as the plantar heater and Von Frey hair tests. Moreover, targeted functional restoration should be tested in studies in which a combination of structures involved in a given function is stimulated. For example, increased efficacy of stimulated corticospinal pathways could be further potentiated by "bottom-up" interventions that involve direct stimulation of propriospinal pathways mediating corticospinal excitation (Yakovenko et al., 2007) or soleus H-reflex operant conditioning (Chen et al., 2006).

The acquisition and maintenance of motor skills undoubtedly require modifications of supraspinal and spinal networks, and the paradigm of Brus-Ramer et al. (2007) offers the possibility to investigate such changes. Inducing and/or strengthening connections between supraspinal and spinal networks via chronic stimulation could have important clinical implications to promote recovery of motor functions after neurotrauma. However, the formation of specific synaptic connections requires an appropriate pattern of activity between presynaptic and postsynaptic neuronal elements. At the level of interacting networks, inducing axonal growth in injured or intact animals without proper behavioral conditioning might subserve no role or might even have detrimental effects by altering the optimal balance required for normal motor function. For instance, what is the functional significance of inducing axonal growth and/or changing transmission efficacy of ipsilateral corticospinal axons to the deep radial nerves in the stimulation-only group [Brus-Ramer et al. (2007), their Fig. $1 B, C$ (http://www.jneurosci.org/cgi/ content/full/27/50/13793/F1)]? In a rehabilitative setting, chronic stimulation paired with appropriate training would be most effective in restoring specific motor functions.

In summary, the paradigm of BrusRamer et al. (2007) offers promising opportunities to restore motor function using activity-dependent processes to strengthen the connectivity between "topdown" and "bottom-up" pathways. Behavioral studies and further examination of plastic changes will be critical for future implementation of this approach in spinal cord injury rehabilitation.

\section{References}

Brus-Ramer M, Carmel JB, Chakrabarty S, Martin JH (2007) Electrical stimulation of spared corticospinal axons augments connections with ipsilateral spinal motor circuits after injury. J Neurosci 27:13793-13801.

Chen Y, Chen XY, Jakeman LB, Chen L, Stokes BT, Wolpaw JR (2006) Operant conditioning of $\mathrm{H}$-reflex can correct a locomotor abnormality after spinal cord injury in rats. J Neurosci 26:12537-12543.

Schomburg ED (1990) Spinal sensorimotor systems and their supraspinal control. Neurosci Res 7:265-340.

Whishaw IQ, Metz GA (2002) Absence of impairments or recovery mediated by the uncrossed pyramidal tract in the rat versus enduring deficits produced by the crossed pyramidal tract. Behav Brain Res 134:323-336.

Yakovenko S, Kowalczewski J, Prochazka A (2007) Intraspinal stimulation caudal to spinal cord transections in rats. Testing the propriospinal hypothesis. J Neurophysiol 97:2570-2574. 